bioRxiv preprint doi: https://doi org/10.1101/2020.05.08.085514; this version posted May 12, 2020. The copyright holder for this preprint

(which was not certified by peer review) is the author/funder, who has granted bioRxiv a license to display the preprint in perpetuity. It is made available under aCC-BY-NC-ND 4.0 International license.

\title{
Silent dissemination of plasmid-borne tigecycline resistance gene tet(X6) in
}

\author{
livestock-associated Acinetobacter towneri
}

Yingying Cheng ${ }^{1 \#}$, Yong Chen ${ }^{1 \#}$, Yang Liu ${ }^{1}$, Jingjie Song ${ }^{1}$, Yuqi Guo ${ }^{1}$, Yanzi Zhou ${ }^{2}$, Tingting Xiao ${ }^{2}$, Shuntian Zhang ${ }^{2}$, Hao Xu ${ }^{2}$, Yunbo Chen ${ }^{2}$, Tongling Shan ${ }^{3}$, Yonghong Xiao ${ }^{2}$, Kai Zhou ${ }^{1 *}$

${ }^{1}$ Shenzhen Institute of Respiratory Diseases, Second Clinical Medical College (Shenzhen People's

Hospital), Jinan University; the First Affiliated Hospital (Shenzhen People's Hospital), Southern

University of Science and Technology, Shenzhen, China;

${ }^{2}$ Collaborative Innovation Center for Diagnosis and Treatment of Infectious Diseases, State Key Laboratory for Diagnosis and Treatment of Infectious Diseases, The First Affiliated Hospital, College of Medicine, Zhejiang University, Hangzhou, China;

${ }^{3}$ Shanghai Veterinary Research Institute, Chinese Academy of Agricultural Sciences, Shanghai, China.

\#These authors contributed equally to this work.

${ }^{*}$ Corresponding author. Mailing address: Dongmen North Road No. 1017, Shenzhen People's Hospital, 518020 Shenzhen, China

E-mail: Kai_Zhou@zju.edu.cn

Telephone: +86-571-2294-4111 
bioRxiv preprint doi: https://doi org/10.1101/2020.05.08.085514: this version posted May $12,2020$. The copyright holder for this preprint

(which was not certified by peer review) is the author/funder, who has granted bioRxiv a license to display the preprint in perpetuity. It is made available under aCC-BY-NC-ND 4.0 International license.

23

24

Abstract:

A tigecycline-resistance gene, tet(X6), was detected on a 159-kb novel plasmid carried by a tigecycline-susceptible livestock-associated Acinetobacter towneri isolate. The genetic context of tet(X6) (DISVsa3-tet(X6)-abh-guaA-ISVsa3) is highly similar with that of the other plasmid-borne tet(X) variants. The 23-Ala residue of the first FAD binding site conferred higher activity to Tet(X6) than the 23-Gly reside conserved in the other plasmid-borne tet(X)s. To our knowledge, this is the first report of tet(X6) carried by the plasmid.

Keywords: plasmid-borne, tet(X6), tigecycline resistance, Acinetobacter towneri

2


Tigecycline is one of the last resorts to treat clinical infections caused by multi-drug resistance (MDR), especially carbapenem-resistant bacteria (1). Tet $(X)$ family contains a series of flavin-dependent monooxygenases, which can degrade tetracycline and tigecycline to 11a-hydroxy-oxytetracycline and 11a-hydroxytigecycline by a similar enzymatic modification pattern, respectively $(2,3)$. The first member of the Tet $(X)$ family was identified in $\operatorname{Tn} 4351$ and Tn4400 carried by the chromosome of anaerobe Bacteroides fragilis (4). Recently, three plasmid-borne tet $(\mathrm{X})$ variants mediating tigecycline resistance, tet $(\mathrm{X} 3)$, tet $(\mathrm{X} 4)$ and tet $(\mathrm{X} 5)$, were consecutively detected in Acinetobacter and Enterobacterales isolates obtained from animals, animal-derived foods, and humans in China (5-7). These findings warn the wide-dissemination possibility of tigecycline resistance in clinical setting mediated by mobile genetic elements. Most recently, tet(X6) was identified on the chromosome of Proteus spp., Myroides spp. and Acinetobacter spp. Strains $(8,9)$, and on integrative and conjugative elements ICEPgs6Chn1 carried by the chromosome of Proteus spp. $(9,10)$. We here first report the detection of a plasmid-borne tet(X6) in a livestock-associated $A$. towneri strain.

PCR was performed with use of universal primers tet(X)-F (5'-TGCTTGAACCTGGTAAGAAG-3') and tet(X)-R (5'-AATGAGCAGCATCGCCAATC-3') to screen tet(X) variants in 290 Acinetobacter spp. strains isolated from livestock stool samples collected in China in 2019. A tet(X6)-positive $A$. towneri strain AT205 recovered from a swine fecal sample was detected. AT205 exhibited resistance to gentamicin and some tetracycline families, but susceptible to tigecycline with the minimum inhibitory concentration (MIC) at $1 \mathrm{mg} / \mathrm{L}$ (Table S1).

To understand the vector of tet(X6), AT205 was sequenced by using Hiseq 4000 system (Illumina, San Diego, US) and PromethION platform (Nanopore, Oxford, UK). Hybrid assembly 
using Unicycler version 0.4.8 (11) resulted in a 2.66-Mb circular chromosome (confirmed by PCR) with GC content of $41.48 \%$ (CP048014), a completed plasmid pAT205 (confirmed by PCR) with a size of $158.867 \mathrm{~kb}$ (CP048015) and three contigs (CP048016-CP048018). The tet(X6) gene was located on the plasmid pAT205 (Figure 1A). This is different with the recent reports that tet(X6) was carried on the chromosome of Proteus spp., Myroides spp. and Acinetobacter spp. strains (8-10). Therefore, this is the fourth plasmid-borne tet $(\mathrm{X})$ variant identified after $\operatorname{tet}(\mathrm{X} 3), \operatorname{tet}(\mathrm{X} 4)$ and tet(X5) $(6,7)$. Notably, Acinetobacter spp. is the common host of tet(X3), tet(X5) and tet(X6) (6-8), suggesting that the genus may be an important reservoir for the plasmid-borne tigecycline-resistance genes.

The genetic context of tet(X6) detected on pAT205 was "AISVsa3-tet(X6)-abh-guaA-ISVsa3". This is highly similar with that of plasmid-borne tet( $\mathrm{X} 3)$, tet $(\mathrm{X} 4)$ and $\operatorname{tet}(\mathrm{X} 5)$ previously reported (5-7) (Figure 1B). It has been shown that the ISVsa3-flanking region is able to become a circular intermediate facilitating the transfer of $\operatorname{tet}(\mathrm{X} 3)$ and $\operatorname{tet}(\mathrm{X} 4)(8)$. Given the similar structure, we suppose that tet(X6) could be mobilized through this unit. pAT205 failed to be transferred to $A$. baumannii ATCC17978 and E. coli EC600 by the conjugation assay, which is due to the lack of functional conjugative system on pAT205 (only one type IV secretory gene was detected).

Blasting the sequence of pAT205 showed only two plasmids with coverage $>35 \%$ (Figure 1A); p19110F47-2 (CP046044) carried by an A. towneri strain (99.98\% identity; 66\% coverage) and plasmid 1 (CP038501) carried by an A. baumannii strain (99.63\% nucleotide identity; 35\% coverage). This suggests that pAT205 is a novel plasmid, and might originate from Acinetobacter spp.. A class 2 integron (50498 bp-67325 bp) region carrying the cassette array of $d f r A 1$, sat2 and aadA1 was identified on pAT205 (Figure S1A). The $3^{\prime}$ conserved segment ( $3^{\prime}$-CS) of the integron 
was consist of five tns genes (tnsA, tnsB, tnsC, tnsD and tnsE) (12), and was disrupted by a 4802-bp fragment encoding a retron-type RNA-directed DNA polymerase and IS66 family mobile elements (Figure S1A). A Tn6205-like structure (22252bp-34180bp) was additionally detected on pAT205 (Figure S1B). Compared with the original structure of Tn6205 (CP003505), 5' and 3' end of the Tn6205-like structure was reversed, and more resistance genes were carried, including a tetracycline resistance regulatory gene tet $(\mathrm{R})$, an efflux pump gene tet $(\mathrm{Y})$ and 2 aminoglycoside resistance genes [aph(4)-Ia and $\operatorname{aac}(3)-I V$ ]. Such plasmid represents a high threat to public health since it confers MDR including the resistance to the last resort antibiotics.

To verify the activity of tet(X6), the fragment between $107 \mathrm{bp}$ upstream and $29 \mathrm{bp}$ downstream of tet(X6) including the predicted promoter was amplified using primers pUC19-tet(X6)-F (5'-cgctgcagGCAATTGACTTTCCGAACGG-3') and p-tet(X6)-R (5'-cgtctagaTTTCTCTTTCATTTCCTCGCC-3'). The resulted product was ligated into pUC19 to construct transformant pUC19-tet(X6) and overexpressed in E. coli DH5 $\alpha$. In parallel, tet(X3) and tet $(\mathrm{X} 4)$ were cloned into pUC19 as positive controls. The tigecycline MIC of the transformant DH5 $\alpha$-pUC19-tet(X6) was $8 \mathrm{mg} / \mathrm{L}$, which was slightly lower than that of tet(X3) and tet(X4) transformants as $16 \mathrm{mg} / \mathrm{L}$ (Figure 2A). The tigecycline MIC of the negative control was $0.125 \mathrm{mg} / \mathrm{L}$ (Table S1). To compare the activity of $\operatorname{Tet}(\mathrm{X} 6)$ with that of $\operatorname{Tet}(\mathrm{X} 3)$ at the low expression level, tet $(\mathrm{X} 6)$ and tet $(\mathrm{X} 3)$ were cloned into pJN105 using the inducible pBAD promoter, respectively. The tigecycline MIC of DH5 $\alpha$-pJN105-tet(X6) and DH5 $\alpha$-pJN105-tet(X3) increased 16-fold and 64-fold respectively (Table S2). This further supports that the activity of Tet(X6) against tigecycline was weaker than that of Tet(X3). 
111 modification to demonstrate the tigecycline inactivation of Tet(X6). The optical density of tet(X)s'

112 clone cultures at $600 \mathrm{~nm}$ were adjusted to 2.0, followed by adding tigecycline into $1 \mathrm{~mL}$ adjusted

113 cultures with final concentration of $2.5 \mathrm{mg} / \mathrm{L}$. After incubation at $37^{\circ} \mathrm{C}$ for 8 hours, the activity of

114 the remaining tigecycline was measured by dropping $100 \mu \mathrm{L}$ supernatant on the LB agar plate

with E. coli ATCC25922 as prey. The diameter of the inhibition zone caused by the supernatant of

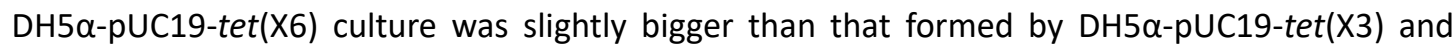
pUC19-tet(X6)-F and tet(X6)-SDM-AtoG-R (5'-CAACAGGCCCTCCACCAATTAT-3') and the right the tigecycline resistance, and the site might be under selection. 
bioRxiv preprint doi: https://doi org/10.1101/2020.05.08.085514; this version posted May 12,2020 . The copyright holder for this preprint

(which was not certified by peer review) is the author/funder, who has granted bioRxiv a license to display the preprint in perpetuity. It is made available under aCC-BY-NC-ND 4.0 International license.

133 tigecycline resistance in clinical setting.

134

\section{Acknowledgments}

This work was supported by the National Key Research and Development Program of China

(2017YFC1200200), Major Infectious Diseases Such as AIDS and Viral Hepatitis Prevention and

coli LHM10-1.

\section{Transparency declarations}

None to declare.

\section{Data availability}

144 The genome sequence of AT205 was submitted to GenBank under the accession numbers

СР048014-СР048018.

\section{References}

147 1. Pankey GA. 2005. Tigecycline. J Antimicrob Chemother 56:470-80.

148 2. Yang W, Moore IF, Koteva KP, Bareich DC, Hughes DW, Wright GD. 2004. TetX is a flavin-dependent monooxygenase conferring resistance to tetracycline antibiotics. J Biol Chem 279:52346-52.

151 3. Moore IF, Hughes DW, Wright GD. 2005. Tigecycline is modified by the flavin-dependent monooxygenase TetX. Biochemistry 44:11829-35.

153 4. Speer BS, Bedzyk L, Salyers AA. 1991. Evidence that a novel tetracycline resistance gene found on two Bacteroides transposons encodes an NADP-requiring oxidoreductase. J 

high-level tigecycline resistance in Escherichia coli. Nat Microbiol. L, Lv Z, Li Y, Pang M, Wang L, Sun Q, Fu Y, Song H, Hao Y, Shen Z, Wang S, Chen G, Wu C, Shen J, Wang Y. 2019. Emergence of plasmid-mediated high-level tigecycline resistance genes in animals and humans. Nat Microbiol. plasmid-mediated tet(X5) gene conferring resistance to tigecycline, eravacycline and

9. Peng K, Li R, He T, Liu Y, Wang Z. 2020. Characterization of a porcine Proteus cibarius strain co-harbouring tet(X6) and cfr. J Antimicrob Chemother. 
177 12. Deng Y, Bao X, Ji L, Chen L, Liu J, Miao J, Chen D, Bian H, Li Y, Yu G. 2015. Resistance integrons: class 1, 2 and 3 integrons. Ann Clin Microbiol Antimicrob 14:45.

13. Grant JR, Stothard P. 2008. The CGView Server: a comparative genomics tool for circular genomes. Nucleic Acids Res 36:W181-4.

14. Sullivan MJ, Petty NK, Beatson SA. 2011. Easyfig: a genome comparison visualizer. Bioinformatics 27:1009-10.

Figure legends:

Figure 1. The structure of tet(X6)-carrying pAT205. (A) The circles from outside to inside depict: (i) the antibiotic resistance genes harbored by pAT205 are marked in red; (ii) the positions of predicted coding sequences transcribed in clockwise and counterclockwise orientation in indigo the GC content plotted against 50\%; (v) GC skew $[(G-C) /(G+C)]$ in a 10000 bp window; (vi) the backbone of pAT205 with size scale in bp. The CGView Server (13) was used to generate the blast results. (B) Comparison of genetic context of plasmid-borne tet(X) variants. Sequence comparison and map generation were performed by using Easyfig 2.2.3 (14). Arrows represent the genes with different directions. Regions of $>80 \%$ homology are shown by grey shading. Genes are colour-coded, depending on functional annotations: red, antimicrobial resistance; green, mobile elements; blue, other functions; orange, hypothetical proteins. 
bioRxiv preprint doi: https://doi.org/10.1101/2020.05.08.085514; this version posted May 12, 2020. The copyright holder for this preprint (which was not certified by peer review) is the author/funder, who has granted bioRxiv a license to display the preprint in perpetuity. It is made available under aCC-BY-NC-ND 4.0 International license.

199 remaining tigecycline in the supernatant of Tet(X)s-overexpressing strains, and ATCC25922 was used as the prey strain. 


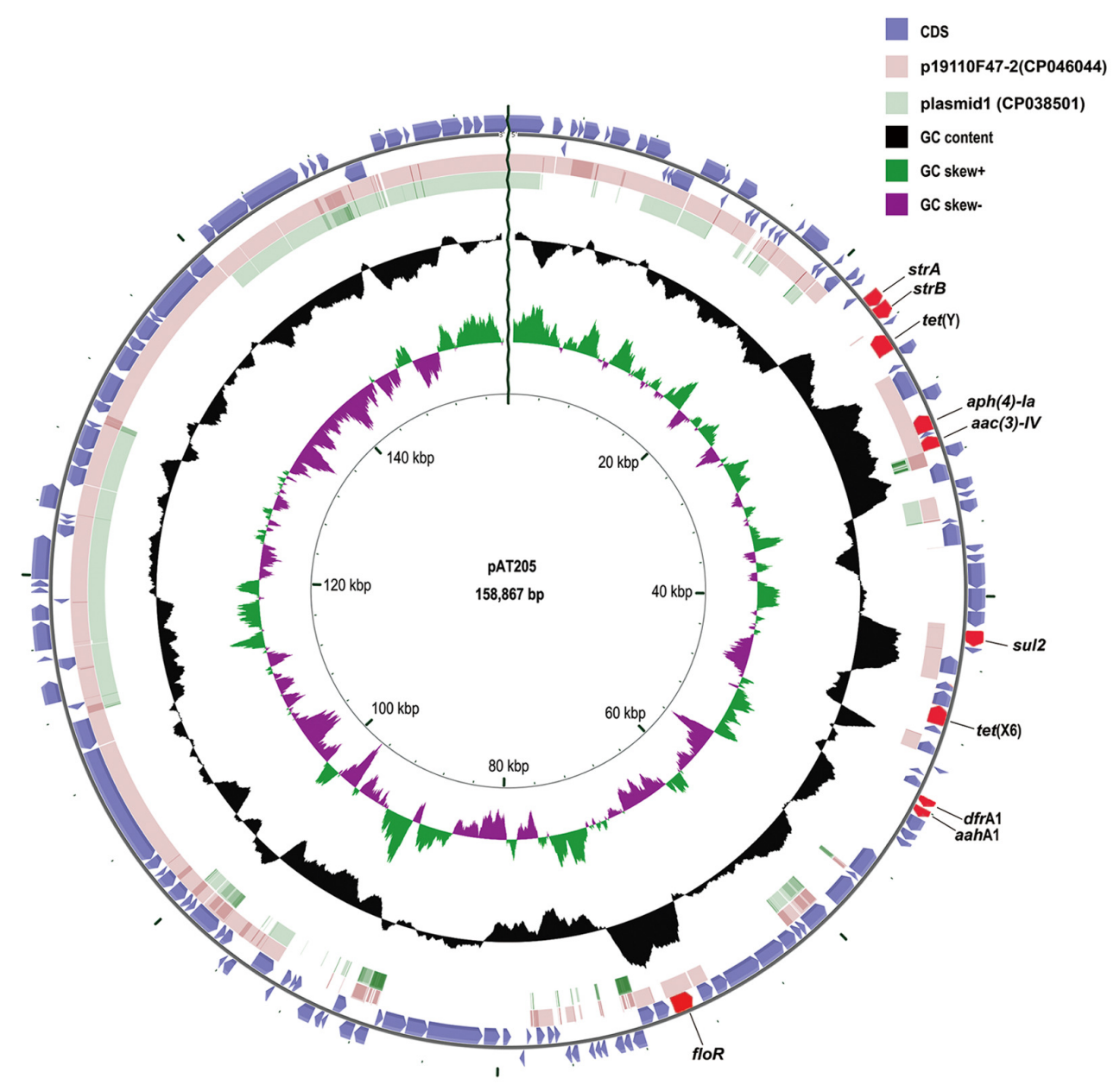

B

E.coli LHM10-1 (CP037909)

A.baumanii 34AB (MK134375)

E.cloi 47EC (MK134376)

A.baumanii $\mathrm{AB} 17 \mathrm{H} 194$ (CP040912)

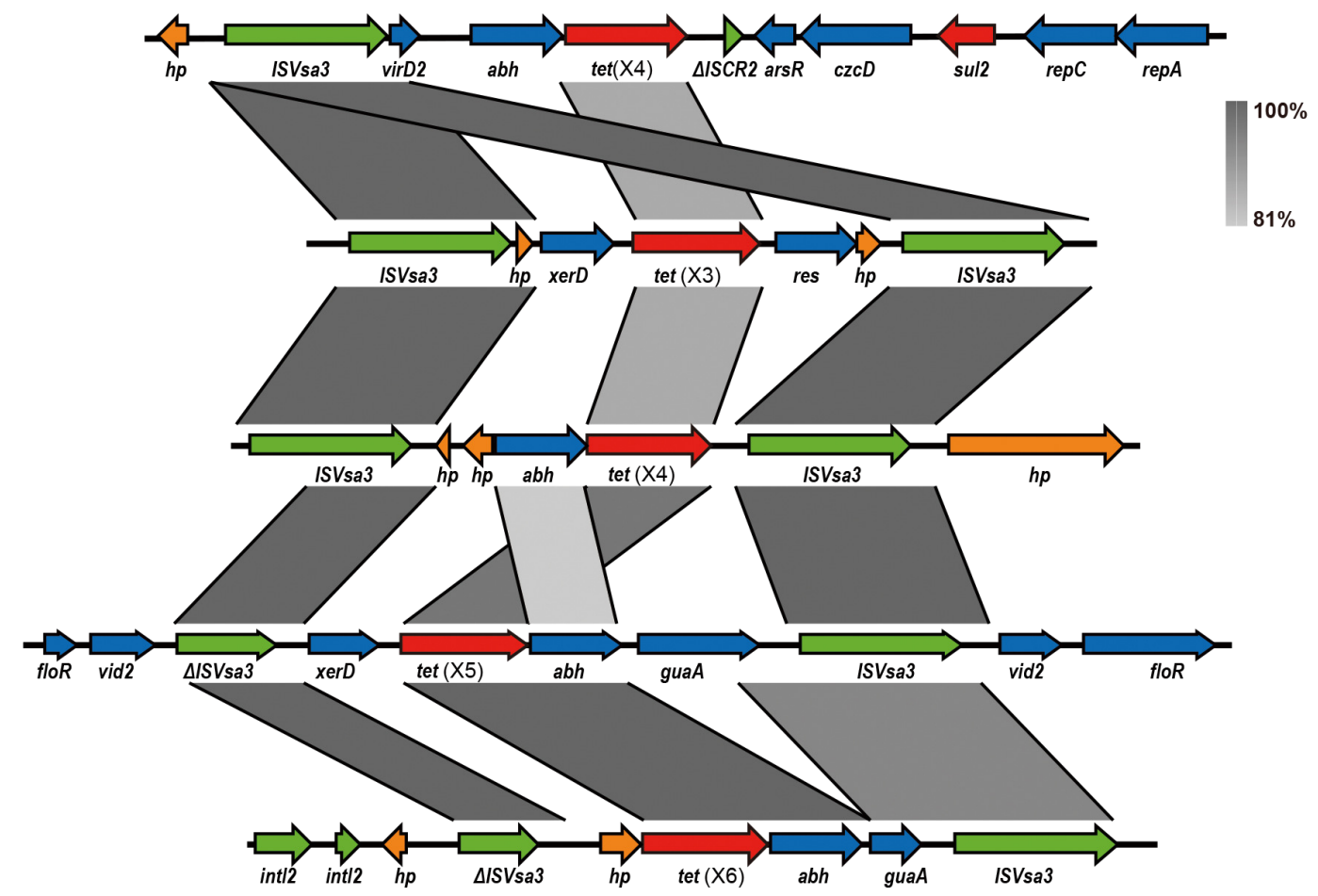



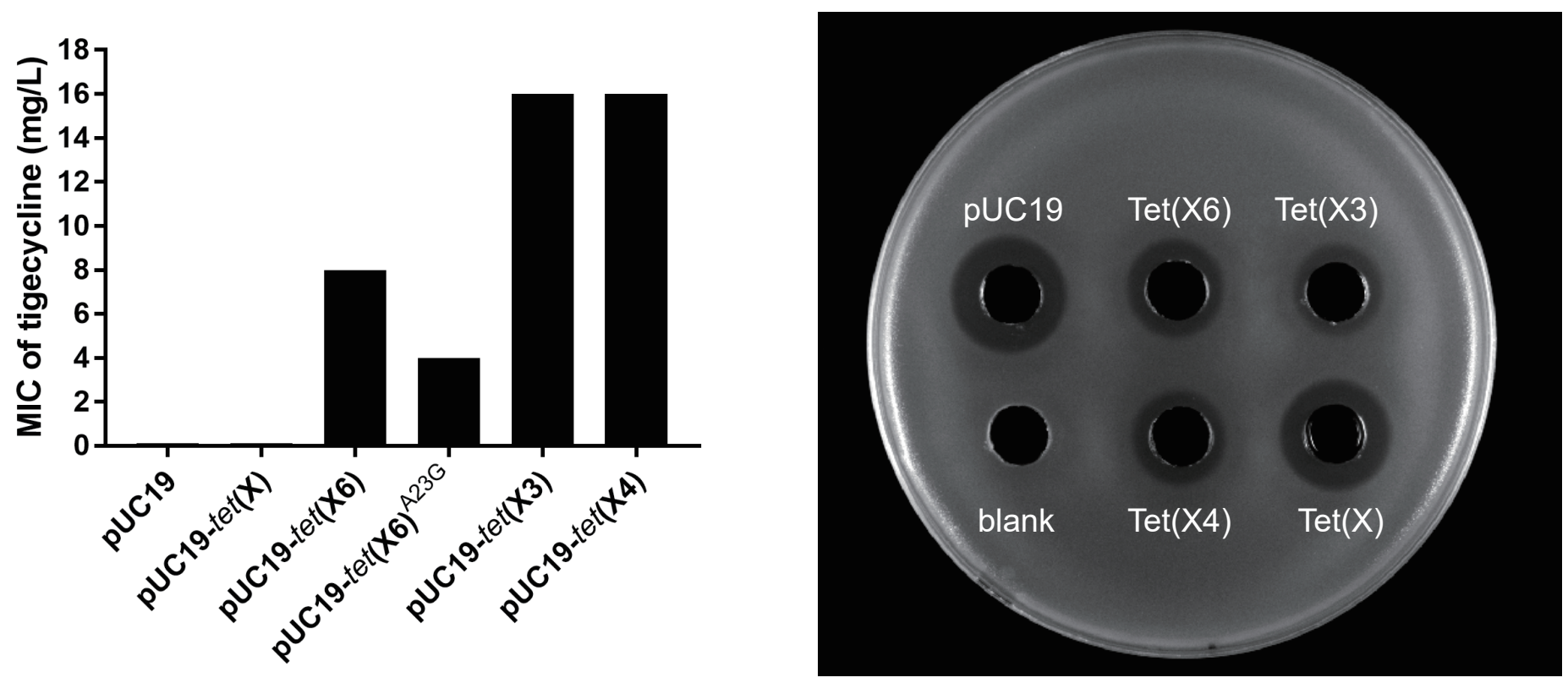